\title{
Anisotropic inflation with derivative couplings
}

\author{
Jonathan Holland, ${ }^{1}$ Sugumi Kanno, ${ }^{2,3}$ and Ivonne Zavala ${ }^{1}$ \\ ${ }^{1}$ Department of Physics, Swansea University, Swansea SA2 8PP, United Kingdom \\ ${ }^{2}$ Department of Theoretical Physics and History of Science, \\ University of the Basque Country 48080 Bilbao, Spain \\ ${ }^{3}$ IKERBASQUE, Basque Foundation for Science, Maria Diaz de Haro 3, Bilbao 48013, Spain
}

(Received 5 December 2017; published 29 May 2018)

\begin{abstract}
We study anisotropic power-law inflationary solutions when the inflaton and its derivative couple to a vector field. This type of coupling is motivated by D-brane inflationary models, in which the inflaton, and a vector field living on the D-brane, couple disformally (derivatively). We start by studying a phenomenological model where we show the existence of anisotropic solutions and demonstrate their stability via a dynamical system analysis. Compared to the case without a derivative coupling, the anisotropy is reduced and thus can be made consistent with current limits, while the value of the slow-roll parameter remains almost unchanged. We also discuss solutions for more general cases, including D-brane-like couplings.
\end{abstract}

DOI: $10.1103 /$ PhysRevD.97.103534

\section{INTRODUCTION}

The most recent data on the cosmic microwave background radiation temperature fluctuations [1,2] favor the simplest inflationary scenario to provide their origin. This simple scenario makes robust predictions for the primordial inhomogeneities of the universe: they are adiabatic, highly isotropic, approximately scale invariant, and nearly Gaussian. In this framework, a single scalar field drives a period of quasi-de Sitter accelerated expansion, while its quantum fluctuations are stretched to observable scales, setting up the initial conditions for structure growth. Most inflationary models are based on scalar field dynamics, where inflation is driven by a single scalar field. The current data have severely constrained some of these models; however, many remain compatible [3]. With more precise observations to come in the future, it will be possible to determine whether more complicated models of inflation are needed, which will narrow down the landscape of observably viable scenarios.

In particular, multifield models of inflation arise naturally in extensions of the simplest cosmological framework, for example, in fundamental theories such as supergravity and string theory. Usually, models with more than one scalar field are considered in these extensions. However, fields with other spins, such as gauge fields, may play an interesting and testable role during inflation as well [4-6].

Published by the American Physical Society under the terms of the Creative Commons Attribution 4.0 International license. Further distribution of this work must maintain attribution to the author(s) and the published article's title, journal citation, and DOI. Funded by SCOAP.
Gauge fields are not commonly considered in the study of inflation, due to the cosmic no-hair conjecture, which states that spacetime rapidly approaches quasi-de Sitter spacetime during inflation. Thus, matter fields satisfying the dominant and strong energy conditions will be rapidly diluted. However, the authors of [7] found the first working model (free from ghosts) of inflation with a vector field that can produce persistent anisotropy in the background spacetime. This brought forward the interesting possibility that light gauge fields may affect cosmological observations by generating some observable amount of statistical anisotropy (for reviews on anisotropic inflation see [4-6]).

Another possibility for generating observable statistical anisotropies in the presence of vector fields is the vector curvaton scenario [8-10]. In this scenario, the inflaton is a scalar driving inflation, while the vector field becomes important after inflation, when it may dominate the universe and imprint its perturbation spectrum before it decays, as in the scalar curvaton scenario (for a review of the vector curvaton see [11]).

In the context of D-brane inflationary models, the inflaton is typically identified with the scalar field parametrizing the transverse fluctuations of the D-brane (that is, its position in the internal compact six-dimensional space). Such a brane features a world-volume two form field $F_{\mu \nu}$, associated with the longitudinal fluctuations of the D-brane. Therefore, it is natural to investigate the role of this brane field in the dynamics of inflation. Indeed, in [12], a D-brane vector curvaton realization was discussed, while in [13] a Wilson line inflationary model was studied with interesting predictions. In this case, it is precisely one of the D-brane vector internal components that drives inflation. 
In a D-brane scenario, the scalar field associated with brane position, identified with the inflaton, and the vector field couple disformally via the Dirac-Born-Infeld (DBI) action describing the D-brane dynamics. ${ }^{1}$ In particular, the gauge kinetic function, $f$, depends on the scalar field $\phi$ and its derivative $X \equiv \frac{1}{2}(\partial \phi)^{2}, f(\phi, X)$. This coupling can thus alter the predictions of the anisotropic background evolution and the predictions for statistical anisotropies. Furthermore, $f(\phi, X)$ represents a general parametrization of a generic inflaton-matter coupling. This has recently been used in studies of the inflationary universe as a cosmological collider [15]. It is thus important to know what effect the derivative coupling has on inflationary evolution. This derivative coupling has also appeared in the recently proposed Effective Field Theory (EFT) of anisotropic inflation [16,17].

Motivated by the D-brane scenario and the more generic nature of a derivative coupling between the inflaton and a vector field (and even more generally, with matter), in this paper we study anisotropic inflation with derivative couplings. We start by considering a phenomenological model where the gauge kinetic function has a monomial dependence on $X$ and exponential dependence on $\phi$. For the power-law cases we consider, no stable solutions exist for when $f(X)$ only. On the other hand, for $f(\phi, X)$, stable anisotropic solutions exist and the anisotropy is considerably reduced in comparison to the nonderivative case. This is interesting in view of the latest constraints on anisotropy $[18,19]$. We next use our general equations to explore more general solutions. Finally we conclude in Sec. V with a discussion of our results and prospects for future work.

\section{SCALAR-VECTOR-TENSOR ACTION WITH GENERAL DERIVATIVE COUPLINGS}

Although we will look only at power-law inflationary solutions, in this section we will keep the discussion as general as possible when presenting the setup and equations of motion. This will be useful to describe a variety of power-law models as we discuss later. In most of the paper we will concentrate on a simple field theory model that will serve to illustrate the consequences of taking derivative couplings into account.

Our starting point is the general scalar-vector-tensor action of the form

$S=\int d^{4} x \sqrt{-g}\left\{\frac{M_{\mathrm{Pl}}^{2}}{2} R-P(\phi, X)-\frac{f^{2}(\phi, X)}{4} F_{\mu \nu} F^{\mu \nu}\right\}$,

where $F_{\mu \nu}=\partial_{\mu} A_{\nu}-\partial_{\nu} A_{\mu}, 2 X=(\partial \phi)^{2}$; thus, we see that the gauge kinetic function depends on both the inflaton, $\phi$,

\footnotetext{
${ }^{1}$ For an example of a theory where two scalar fields couple disformally via the DBI action, see [14].
}

and its derivative, $X$. This action is motivated from D-brane actions in string theory models of inflation, where $P(\phi, X)$ and $f(\phi, X)$ take very specific forms and arise from the DBI action (see [12] for details). Here we keep these functions general, in order to cover other possibilities. ${ }^{2}$

The equations of motion derived from (1) are given by

$$
\begin{aligned}
& R_{\mu \nu}-\frac{1}{2} g_{\mu \nu} R=8 \pi G\left(T_{\mu \nu}^{A}+T_{\mu \nu}^{\phi}\right), \\
& \frac{1}{\sqrt{-g}} \partial_{\mu}\left[\sqrt{-g}\left(\frac{F^{2}}{2} f f_{X}+P_{X}\right) \partial^{\mu} \phi\right]=\frac{F^{2}}{2} f f_{\phi}+P_{\phi}, \\
& \partial_{\mu}\left[\sqrt{-g} f^{2}(\phi, X) F^{\mu \nu}\right]=0,
\end{aligned}
$$

where $8 \pi G=M_{\mathrm{Pl}}^{-2}$ is the reduced Planck mass and we have denoted the derivatives as $f_{i}=\partial_{i} f$ and similarly for $P$, for $i=\phi, X$. The energy-momentum tensors for the vector and the scalar fields are given by

$$
\begin{aligned}
T_{\mu \nu}^{\phi} & =\partial_{\mu} \phi \partial_{\nu} \phi\left(\frac{1}{2} f f_{X} F^{2}+P_{X}\right)-g_{\mu \nu} P, \\
T_{\mu \nu}^{A} & =f^{2}\left[F_{\nu}{ }^{\alpha} F_{\mu \alpha}-g_{\mu \nu} \frac{F^{2}}{4}\right] .
\end{aligned}
$$

We are interested in anisotropic solutions, and therefore, without loss of generality, we consider the following anisotropic metric:

$d s^{2}=-d t^{2}+e^{2 \alpha(t)}\left[e^{-4 \sigma(t)} d x^{2}+e^{2 \sigma(t)}\left(d y^{2}+d z^{2}\right)\right]$,

where $e^{\alpha(t)}$ is identified with the isotropic scale factor, and $e^{\sigma(t)}$ characterizes the anisotropy. Furthermore, we use gauge invariance to choose $A_{0}=0$, and, for concreteness, we consider homogeneous fields of the form $[7,22]$

$$
\phi=\phi(t), \quad A_{\mu}=(0, v(t), 0,0) .
$$

With these Ansätze, the equation of motion for the vector field takes the simple form

$$
\frac{d}{d t}\left[f^{2} e^{\alpha+4 \sigma} \dot{v}\right]=0,
$$

which can readily be solved to give

$$
f^{2} e^{\alpha+4 \sigma} \dot{v}=p_{A}
$$

where $p_{A}$ is a constant of integration. Since $-\dot{v}=F_{x 0}=E_{x}$, $p_{A}$ is the electric field modulated by the expansion of the universe.

\footnotetext{
${ }^{2}$ Anisotropic inflationary solutions with a DBI kinetic term for the scalar field and a pure inflaton-dependent gauge kinetic function $f$ were considered in [20]. More general forms for $P(\phi, X)$ were further considered in [21].
} 
The Einstein equations, on the other hand, can be arranged into the following set of equations:

$$
\begin{aligned}
\ddot{\alpha}= & -3 \dot{\alpha}^{2}+\frac{1}{6 M_{\mathrm{Pl}}^{2}}\left[6 P+f^{2} \dot{v}^{2} e^{-2 \alpha+4 \sigma}\right. \\
& \left.+3 \dot{\phi}^{2}\left(P_{X}-\frac{f_{X}}{f} f^{2} \dot{v}^{2} e^{-2 \alpha+4 \sigma}\right)\right], \\
\ddot{\sigma}= & -3 \dot{\alpha} \dot{\sigma}+\frac{f^{2} \dot{v}^{2}}{3 M_{\mathrm{Pl}}^{2}} e^{-2 \alpha+4 \sigma}, \\
\dot{\alpha}^{2}= & \dot{\sigma}^{2}+\frac{1}{3 M_{\mathrm{Pl}}^{2}}\left[P+\frac{f^{2} \dot{v}^{2}}{2} e^{-2 \alpha+4 \sigma}\right. \\
& \left.+\dot{\phi}^{2}\left(P_{X}-\frac{f_{X}}{f} f^{2} \dot{v}^{2} e^{-2 \alpha+4 \sigma}\right)\right],
\end{aligned}
$$

where we have used (10). Finally, the equation of motion for the scalar field becomes

$$
\begin{aligned}
\ddot{\phi} & {\left[-\dot{\phi}^{2} P_{X X}+P_{X}-f^{2} \dot{v}^{2} e^{-2 \alpha+4 \sigma}\left(\frac{f_{X}}{f}+3 \frac{f_{X}^{2}}{f^{2}} \dot{\phi}^{2}-\frac{f_{X X}}{f} \dot{\phi}^{2}\right)\right] } \\
& +\dot{\phi}\left[\dot{\phi} P_{X \phi}+3 \dot{\alpha} P_{X}+f^{2} \dot{v}^{2} e^{-2 \alpha+4 \sigma}\right. \\
& \left.\times \frac{f_{X}}{f}\left(\dot{\phi}\left(3 \frac{f_{\phi}}{f}-\frac{f_{X \phi}}{f_{X}}\right)+4 \dot{\sigma}+\dot{\alpha}\right)\right] \\
& +P_{\phi}-\frac{f_{\phi}}{f} f^{2} \dot{v}^{2} e^{-2 \alpha+4 \sigma}=0
\end{aligned}
$$

From these equations, it is easy to recover the various examples studied in the literature, for which $f_{X}=0$ [7,20-23].

In what follows we use these equations to look for stable anisotropic solutions. We start by looking at a phenomenological example that serves as a prototype to understand the effect of the derivative coupling between the inflaton and the vector field, and then we explore more general cases.

\section{ANISOTROPIC POWER-LAW INFLATION WITH DERIVATIVE COUPLINGS}

In this section we start the analysis of power-law anisotropic inflation with derivative couplings, providing the first explicit example of the situation described in the EFT description of [16]. We start with a canonically normalized inflaton,

$$
P(\phi, X)=\frac{1}{2}(\partial \phi)^{2}+V(\phi)=X+V,
$$

and thus replace, $P_{\phi}=V_{\phi}, P_{X}=1$ in the equations of motion above, (11)-(14). Note that Eqs. (11) and (13) depend only on the derivative of $f$ with respect to $X$. One then immediately sees that a suitable choice of functional form is given by setting

$$
X \frac{f_{X}}{f}=-n,
$$

where $n=$ const. This has the solution

$$
f(X, \phi)=(-X)^{-n} g(\phi),
$$

for some function $g(\phi)$. On the other hand, we can also see that a suitable choice of $\phi$ dependence is given when $f_{\phi} / f=$ const, that is, an exponential dependence. So we find that a suitable Ansatz for the gauge kinetic function's dependence on the scalar and its derivative is given $b^{3}$ :

$$
f(\phi, X)=M_{\mathrm{Pl}}^{4 n} f_{0} \frac{e^{\frac{\rho}{M_{\mathrm{Pl}}} \phi}}{(-X)^{n}} .
$$

In addition to this, we also consider an exponential potential for the scalar field

$$
V(\phi)=V_{0} e^{\frac{\lambda}{M_{\mathrm{Pl}}} \phi} .
$$

We are now ready to look for power-law solutions of the form

$$
\begin{aligned}
\alpha & =\zeta \log \left(M_{\mathrm{Pl}} t\right), \quad \sigma=\eta \log \left(M_{\mathrm{Pl}} t\right), \\
\frac{\phi}{M_{\mathrm{Pl}}} & =\xi \log \left(M_{\mathrm{Pl}} t\right)+\phi_{0} .
\end{aligned}
$$

Using this Ansatz with the Hamiltonian constraint (13), we obtain the conditions

$$
\lambda \xi=-2, \quad \rho \xi+2 \zeta+2 \eta+2 n=1 .
$$

We arrive at these two conditions (21) by requiring that, after substitution of (20) into (11)-(14), powers in $t$ balance in all equations (i.e., we end up with equations of the form $C t^{n}=K t^{n}$ where $C, K$ are independent of $t$ ). The remaining conditions (below) come from ensuring that $C=K$ in (11)-(14), i.e., that the equations are satisfied (the amplitudes balance) after substitution of (20). For the amplitudes to balance in (13) (the Hamiltonian constraint), it is required that

$$
-\zeta^{2}+\eta^{2}+\frac{1}{6} \xi^{2}+\frac{1}{3} u+(1-4 n) 4^{-n} \xi^{4 n} \frac{w}{6}=0,
$$

where we have defined $u, w$ as

$$
u=\frac{V_{0}}{M_{\mathrm{Pl}}^{4}} e^{\lambda \phi_{0}}, \quad w=\frac{p_{A}^{2}}{M_{\mathrm{Pl}}^{4}} f_{0}^{-2} e^{-2 \rho \phi_{0}} .
$$

\footnotetext{
${ }^{3}$ In a string theory scenario, the effective four-dimensional (4D) action can be written in terms of the 4D $M_{\mathrm{Pl}}$, which would be a function of the string scale and coupling, as well as the compactification volume.
} 
From the equation for the scale factor (11) we then obtain

$$
3 \zeta^{2}-\zeta-u+4^{-n}(6 n-1) \xi^{4 n} \frac{w}{6}=0
$$

Similarly from the anisotropy equation (12) we get

$$
-\eta(3 \zeta-1)-4^{-n} \xi^{4 n} \frac{w}{3}=0 .
$$

Finally from the equation for the inflaton we obtain

$$
\begin{aligned}
& -\xi+3 \zeta \xi+\lambda u+4^{-n} \xi^{4 n-1} w[2 n(\zeta+4 \eta+4 n-1) \\
& +\rho \xi(4 n-1)]=0 .
\end{aligned}
$$

Using these equations, we can solve for $u$ and $w$ to get

$$
u=-\zeta+3 \zeta^{2}+\frac{1}{2} \eta-3 n \eta-\frac{3}{2} \zeta \eta+9 n \zeta \eta,
$$

$w=3 \eta 4^{n} \xi^{-4 n}(3 \zeta-1)$.

Substituting these into the inflaton equation (26), and using the constraints for $\xi$ and $\eta$ from (21), gives

$$
\begin{gathered}
(-1+3 \zeta)\left[8+\lambda^{2}(1-6 \zeta+2 n(-1+9 \zeta(-1+2 n+2 \zeta)))\right. \\
\left.-4 \lambda \rho(-2+3 \zeta+3 n(1+3 \zeta))+12 \rho^{2}\right]=0 .
\end{gathered}
$$

In contrast with [22], we now obtain a cubic, rather than a quadratic equation for $\zeta$. As in [22], we have the solution $\zeta=\frac{1}{3}$ which gives $u=w=0$, implying that there is no anisotropy and no potential driving inflation. We hence discard this solution and focus on the other two:

$$
\zeta_{+}=\frac{A+\sqrt{B}}{72 n \lambda^{2}}, \quad \zeta_{-}=\frac{A-\sqrt{B}}{72 n \lambda^{2}},
$$

where

$$
A=6 \lambda^{2}+18 n \lambda^{2}-36 n^{2} \lambda^{2}+12 \lambda \rho+36 n \lambda \rho,
$$

and

$$
B=A^{2}-144 n \lambda^{2}\left(8+\lambda^{2}-2 n \lambda^{2}+8 \lambda \rho-12 n \lambda \rho+12 \rho^{2}\right) .
$$

These solutions trivially satisfy (22), and it is important to remember that they are constrained from the requirement that $w, u$ must be positive by definition, (23).

To look for inflationary solutions we define the average slow-roll parameter, $\epsilon$ in terms of the Hubble parameter defined by $H=\dot{\alpha}$, as

$$
\epsilon \equiv-\frac{\dot{H}}{H^{2}}=\frac{1}{\zeta} .
$$

Hence there are two branches of solutions for $\epsilon$ corresponding to $\zeta_{ \pm}$. To have inflation, we need $\epsilon \ll 1$; that is, we are looking for regions in the parameter space where $\zeta_{ \pm} \gg 1$.

The anisotropy is characterized by

$$
\frac{\Sigma}{H} \equiv \frac{\dot{\sigma}}{\dot{\alpha}}=\frac{\eta}{\zeta}
$$

where $\eta$ is given by

$$
\eta=\frac{1}{2}+\frac{\rho}{\lambda}-\zeta-n
$$

As for $\epsilon$, there are two possible branches of solutions, associated with $\zeta_{ \pm}$.

Let us now discuss two cases of interest. First, $\rho=0$, which corresponds to a gauge kinetic function that depends only on the derivative of the scalar field. And second, $\rho \neq 0$, when it depends on both.

a. Shift symmetric coupling, $\rho=0$. A purely shift symmetric coupling of the inflaton with the vector field arises for $\rho=0$ [see (18)]. This type of coupling of the inflaton to matter was considered recently in [15]. For inflationary solutions to arise, we need $\zeta \gg 1$. Moreover, the solutions should satisfy $u, w>0$ [see (27)]. In terms of $\zeta$ (with $\rho=0), w$ and $u$ are given by

$$
\begin{array}{r}
w=-3 \times 2^{-2 n-1}(3 \zeta-1)\left(-\frac{1}{\lambda}\right)^{-4 n}(2 \zeta+2 n-1), \\
u=-\frac{1}{4}(2 n-1)(3 \zeta-1)(6 \zeta+6 n-1) .
\end{array}
$$

Therefore, for $w, u$ to be positive, $n$ must be negative and $|n| \gg 1$, so that $n<1 / 2-\zeta$. From the expressions for $\zeta_{ \pm}$ (29) we see that for $|n| \gg 1, \zeta_{ \pm} \sim \frac{n}{2}(-1 \pm 1){ }^{4}$ Thus in principle there are anisotropic inflationary solutions that satisfy all necessary conditions for sufficiently large $|n|$. For example, $n=-10^{5}, \lambda=1$, gives $\epsilon \sim 10^{-4}, \Sigma / H \sim 10^{-9}$, as required by current bounds $[18,19]$. Note that requiring sufficiently small anisotropy requires very large values of $|n|$. However, we find no stable solutions when $\rho=0 .^{5}$

$b$. More general coupling, $\rho \neq 0$. Let us now consider the case when the gauge kinetic function depends on both the inflaton and its derivative, that is, $\rho \neq 0$. As before, inflationary solutions require $\zeta \gg 1$. Furthermore, the conditions for a positive $w$ (and $u$ ) can be obtained by looking at (27), which takes the form

\footnotetext{
${ }^{4}$ Notice that to see that $u$ is positive in this limit (as required) one needs to include the next to leading order term in the large $n$ expansion for $\zeta_{ \pm}$.

${ }^{5}$ This has been confirmed recently in [24].
} 

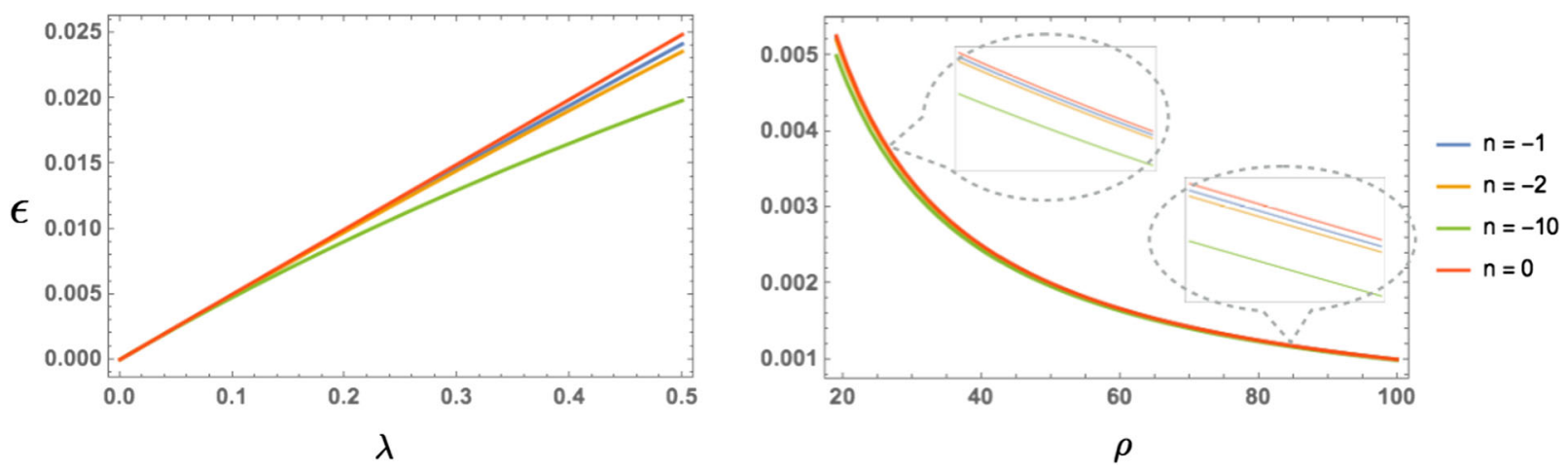

FIG. 1. In these plots we show how the slow-roll parameter, $\epsilon$, varies with $\lambda$ (for $\rho=20$ ) and $\rho$ (for $\lambda=0.1$ ), for the values of $n$ shown.

$$
w=-3 \times 2^{-2 n}(3 \zeta-1)\left(-\frac{1}{\lambda}\right)^{-4 n}\left(\zeta+n-\frac{\rho}{\lambda}-\frac{1}{2}\right) .
$$

Therefore for sufficiently large $\zeta, w$ can be positive for positive or negative $n$ and large values of $\rho / \lambda$, which is also required to obtain large values of $\zeta$ [see Eq. (29)]. In the limit $\rho / \lambda \gg 1$, the solutions $\zeta_{ \pm}$become

$$
\zeta_{ \pm} \simeq \frac{\rho\left(1+3 n \pm \sqrt{(3 n-1)^{2}-\frac{8 n}{\rho^{2}}}\right)}{6 n \lambda} .
$$

Examining this, we see that for $n>0$, the numerator is always positive since $(1+3 n)>\sqrt{(3 n-1)^{2}-(8 n) / \rho^{2}}$ meaning $\zeta_{ \pm}$are both positive. Similarly for $n<0$, we see that $|1+3 n|<\sqrt{(3 n-1)^{2}-(8 n) / \rho^{2}}$, which tells us that $\zeta_{+}$is negative while $\zeta_{-}$is positive. That is, in this limit, there are positive solutions for both $\zeta_{ \pm}$for positive $n$, while for negative $n$ only one solution is positive. We are also interested in small anisotropy, $\Sigma / H \ll 1$ (33), where in terms of the parameters we have

$$
\frac{\Sigma}{H}=\frac{1}{\zeta}\left(\frac{\rho}{\lambda}+\frac{1}{2}-n-\zeta\right)
$$

which further selects the appropriate solution for $\zeta^{6}$ With these conditions, one can check that there is a range of values for which anisotropic solutions exist for $n>0$. A stability analysis shows that for these cases, both the isotropic and the anisotropic solutions are attractors. Therefore the evolution of the system depends on the initial conditions. Since we are interested in the case where the anisotropic solution is the only attractor, in what follows we focus our search for anisotropic stable solutions to the case $n<0$.

In Fig. 1 we show the behavior of the slow-roll parameter, $\epsilon$, as a function of the parameters $\lambda$ and $\rho$ for different values of $n$. As one can see, the slow-roll

\footnotetext{
${ }^{6}$ We focus only on solutions with $|n| \geq 1$, which guarantees real values for $w$ in the $\lambda>0$ we are interested in.
}

parameter $\epsilon$ decreases very slightly as the magnitude of $n$ increases. Conversely, as can be seen in Fig. 2 the anisotropy, $\Sigma / H$, can be reduced by the introduction of a derivative coupling: the greater the magnitude of $n$, the smaller the magnitude of anisotropy.

We can understand the decrease in the anisotropy as follows. Since we are only interested in solutions where the anisotropic point is a single attractor (and since the only observable anisotropic effects come from the final value of the anisotropy), we do not have to worry about the initial value for the gauge field. If the anisotropy converges to a number, its final value is given by the ratio of energy density of the vector field to that of the scalar field [25]. From Eq. (12), we can define this ratio $\mathcal{R}$ as

$$
\frac{\Sigma}{H} \simeq \frac{2}{3} \mathcal{R}, \quad \mathcal{R}=\frac{\rho_{v}}{V(\phi)} \sim \frac{\frac{f^{2}}{2} \dot{v}^{2} e^{-2 \alpha+4 \sigma}}{V(\phi)} .
$$

Using (10), (18), and (19), this ratio can be written as

$$
\mathcal{R} \sim \dot{\phi}^{4 n} e^{-2 \rho \phi-4 \alpha-4 \sigma-\lambda \phi} \sim \xi^{4 n} t^{-2 \rho \xi-4 \zeta-4 \eta-\lambda \xi-4 n},
$$

where in the second expression we used (20). Furthermore, using the conditions (21), we find that the ratio becomes a constant given by

$$
\mathcal{R} \sim \xi^{4 n}=\frac{1}{\xi^{4|n|}}, \quad n<0,
$$

and thus we see why the anisotropy decreases with $|n|$ in the case with derivative couplings. Since $\xi^{4 n}$ is coming from the energy density of the vector field, we see that the anisotropy is reduced because the energy density of the vector field becomes small during inflation.

\section{A. Stability of the anisotropic solutions}

We now study the stability of the solutions above using a dynamical system analysis. For this, we define the dimensionless variables: 

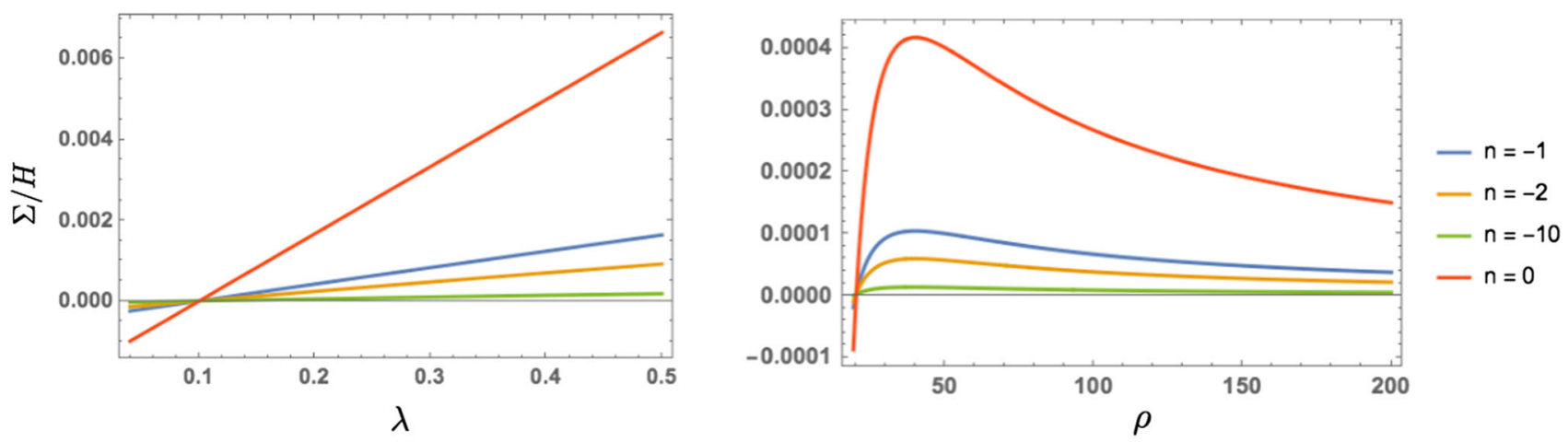

FIG. 2. In these plots we show how the anisotropy, $\frac{\Sigma}{H}$, varies with $\lambda$ (for $\rho=20$ ) and $\rho$ (for $\lambda=0.1$ ), for negative values of $n$ as shown.

$W=\frac{\dot{\sigma}}{\dot{\alpha}}, \quad Y=\frac{1}{M_{\mathrm{Pl}}} \frac{\dot{\phi}}{\dot{\alpha}}, \quad Z=\frac{f(\phi, X) e^{-\alpha+2 \sigma}}{M_{\mathrm{Pl}}} \frac{\dot{v}}{\dot{\alpha}}$,

where we use the $e$-fold number as time coordinate, $d \alpha=$ $\dot{\alpha} d t$. Using these variables, the Hamiltonian constraint (13) can be written as

$$
-\frac{V}{M_{\mathrm{Pl}}^{2} \dot{\alpha}^{2}}=3\left(W^{2}-1\right)+\frac{Y^{2}}{2}+\frac{Z^{2}}{2}(1-4 n) .
$$

Since the inflationary potential is positive definite, we immediately see that (44) implies

$$
W^{2}+\frac{Y^{2}}{6}+\frac{Z^{2}}{6}(1-4 n)<1
$$

In terms of the variables (43), the slow-roll parameter $\epsilon$, becomes

$$
\epsilon=3 W^{2}+\frac{Y^{2}}{2}+\frac{Z^{2}}{3}(1-3 n)
$$

Using the Hamiltonian constraint (44), the equations of motion in terms of (43) can be written as

$\frac{d W}{d \alpha}=\frac{1}{3} Z^{2}(W+1-3 n W)+W\left(3\left(W^{2}-1\right)+\frac{1}{2} Y^{2}\right)$

$$
\begin{aligned}
\frac{d Y}{d \alpha}= & \frac{1}{6} Y\left\{18 W^{2}+3 Y^{2}+2 Z^{2}-6 n Z^{2}+3 C(Y, Z)\right. \\
& \times\left[-4 n Z^{2}(1+4 W)+Y\left(-6 Y+6 \lambda\left(W^{2}-1\right)+\lambda Y^{2}\right.\right. \\
& \left.\left.\left.-Z^{2}(-1+4 n)(\lambda+2 \rho)\right)\right]\right\},
\end{aligned}
$$

$$
\begin{aligned}
\frac{d Z}{d \alpha}= & \frac{1}{6} Z\left\{-12-12 W+18 W^{2}+3 Y^{2}+2 Z^{2}-6 n Z^{2}\right. \\
& -6 \rho Y-6 n C(Y, Z)\left[4 n Z^{2}(1+4 W)\right. \\
& -Y\left(-6 Y+6 \lambda\left(W^{2}-1\right)+\lambda Y^{2}\right. \\
& \left.\left.\left.-Z^{2}(4 n-1)(2 \rho+\lambda)\right)\right]\right\},
\end{aligned}
$$

where

$$
C(Y, Z)=\frac{1}{Y^{2}+2 n Z^{2}(1-4 n)} .
$$

We can now find the fixed points of the system by setting $d W / d \alpha=d Y / d \alpha=d Z / d \alpha=0$. From (47), we find

$$
Z^{2}=\frac{3 W\left(-6+6 W^{2}+Y^{2}\right)}{2(-1-W+3 n W)}
$$

These equations are solved numerically for suitable values of the parameters $\lambda, \rho$, and $n$ (chosen so that $w$ is positive) such that $W, Y$, and $Z$ are all nonzero and real. To choose appropriate solutions, we perform a linear stability analysis. The isotropic fixed point solution is located at $W=Z=0$, $Y=-\lambda$, corresponding with the coupling $f(\phi, X)$ being switched off. The linearized equations of motion around this point reduce to

$$
\begin{aligned}
\frac{d \delta W}{d \alpha} & =\left(\frac{1}{2} \lambda^{2}-3\right) \delta W, \\
\frac{d \delta Y}{d \alpha} & =\left(\frac{1}{2} \lambda^{2}-3\right) \delta Y, \\
\frac{d \delta Z}{d \alpha} & =\left[\left(\frac{1}{2}-n\right) \lambda^{2}+\rho \lambda-2\right] \delta Z .
\end{aligned}
$$

When $\lambda$ is small, the left-hand sides of these equations are all negative (corresponding to the isotropic fixed point being an attractor solution) if $\lambda^{2}(1-2 n)+2 \rho \lambda<4$. If, however, $\lambda^{2}(1-2 n)+2 \rho \lambda>4$, the isotropic fixed point is unstable. Since we are searching for anisotropic solutions, this is the parameter space we want to consider: we require that $\rho>\frac{2}{\lambda}-\frac{\lambda}{2}(1-2 n)$.

Now, we look at two explicit examples to demonstrate that stable derivative anisotropic solutions can be found with small but nonzero anisotropy in agreement with recent data. Consider first the case with $n=-1, \lambda=0.1$, 
and $\rho=20$, which has a fixed point at $(W, Y, Z)=$ $\left(3.08249 \times 10^{-6},-9.92559 \times 10^{-2}, \pm 5.26275 \times 10^{-3}\right)$. Linearization around this point gives

$$
\begin{gathered}
\frac{d \delta W}{d \alpha}=-2.99504 \delta W-3.05955 \times 10^{-7} \delta Y \\
\pm 3.50854 \times 10^{-3} \delta Z \\
\frac{d \delta Y}{d \alpha}=-2.29684 \times 10^{-3} \delta W-3.08768 \delta Y \pm 0.87150 \delta Z
\end{gathered}
$$

$$
\begin{aligned}
\frac{d \delta Z}{d \alpha}= & \mp 1.07688 \times 10^{-2} \delta W \mp 0.434252 \delta Y \\
& +9.26389 \times 10^{-2} \delta Z,
\end{aligned}
$$

where the change in signs is due to choosing either the positive or the negative $Z$ solution. This system has eigenvalues $\left(-2.99504,-2.96384,-3.11922 \times 10^{-2}\right)$ whose real parts are all negative. This system has an average slow-roll parameter of $\epsilon=4.96279 \times 10^{-3}$ [from both (46) and $\epsilon_{-}$in (32)] and anisotropy $\Sigma / H=3.08249 \times 10^{-6}$, which is, however, too large compared to current data $[18,19]$. As a reference, from (23), we can also evaluate the constant of integration for the vector field defined in (10) in terms of $M_{\mathrm{Pl}}, f_{0}$, and $\phi_{0}{ }^{7}$ We can compare this solution with the nonderivative stable one $\lambda=0.1, \rho=50, n=0$. In that case, $\Sigma / H=4 \times 10^{-4}$, and thus we clearly see that the derivative coupling decreases the level of anisotropy.

As a second example we take $n=-2, \lambda=0.01$, and $\rho=200$. This has a stable fixed point at $(W, Y, Z)=$ $\left(2.97549 \times 10^{-10},-9.99875 \times 10^{-3}, \pm 5.17484 \times 10^{-5}\right)$. Linearization of the $W, Y, Z$ equations around this point gives the equations

$$
\begin{gathered}
\frac{d \delta W}{d \alpha}=-2.99995 \delta W-2.97512 \times 10^{-12} \delta Y \\
\pm 3.44989 \times 10^{-5} \delta Z, \\
\frac{d \delta Y}{d \alpha}=-4.28930 \times 10^{-6} \delta W-3.00295 \delta Y \pm 0.145051 \delta Z,
\end{gathered}
$$

$$
\begin{aligned}
\frac{d \delta Z}{d \alpha}= & \mp 1.03586 \times 10^{-4} \delta W \mp 7.25192 \times 10^{-2} \delta Y \\
& +3.00290 \times 10^{-3} \delta Z,
\end{aligned}
$$

where the change in signs is due to choosing either the positive or the negative $Z$ solution, respectively.

\footnotetext{
${ }^{7}$ For example, for $\left(\phi_{0}, f_{0}\right)=(-1,1)$, we find $p_{A}= \pm 4.4 \times$ $10^{-7} M_{\mathrm{Pl}}^{2}$. Inverting (40), we can also find the value of $\dot{v}$ in terms of all the parameters of the model, $\dot{v} \sim\left(\mathcal{R} V 2 f^{-2} e^{2 \alpha-4 \sigma}\right)^{1 / 2}$.
}

The eigenvalues for this set of equations are $(-2.99995$, $\left.-2.99945,-5.00566 \times 10^{-4}\right)$. The eigenvalues' real parts are all negative, and hence this fixed point is stable. Therefore, this corresponds to a stable solution that produces anisotropy during inflation. Using (46), we find the slow-roll parameter to be $\epsilon=4.99938 \times 10^{-5}$ matching perfectly with the $\zeta_{-}$solution in (29), which gives the slow-roll parameter (for $\lambda, \rho, n$ given above) as $\epsilon_{-}=$ $4.99938 \times 10^{-5}$. The average anisotropy is given by $\Sigma / H=2.97549 \times 10^{-10}$ and is thus consistent with observations $[18,19]$. In addition, $w$ is positive and real. We can compare this solution with the nonderivative case of [22] with a slight change in the parameters. In that case, a stable anisotropic solution can be found for $\lambda=0.01, \rho=500$ (and, of course, $n=0$ ), so it is of the same order of magnitude as the present case. For that solution, the anisotropy turns out to be $\Sigma / H=4 \times 10^{-6}$ and thus in tension with current data. Again, we see that a derivative coupling helps to bring apparently excluded solutions back into agreement with observations.

\section{MORE GENERAL SOLUTIONS}

In the previous section we explored a suitable generalization of the nonderivative anisotropic power-law inflation studied in [22] where the gauge kinetic function has a monomial dependence on the inflaton's velocity. Our general equations, however, allow for an easy exploration of other interesting possibilities. One such possibility is the case of DBI inflation [26], where the inflaton can be identified with a D-brane position or a Wilson line. In any case, the vector field featuring on the inflationary D-brane may give rise to anisotropic solutions. In this model, the scalar action and gauge kinetic function are given by [12]

$$
P(\phi, X)=\frac{2 X \gamma}{\gamma+1}+V(\phi), \quad f(\phi, X)=\gamma^{1 / 2}, \quad \gamma=\frac{1}{\sqrt{1+2 h X}},
$$

where $h(\phi)$ is a function of the scalar field only (the warp factor associated with the 10-dimensional geometry where the brane is moving). We see that in the nonrelativistic case, when $\gamma \rightarrow 1$, the scalar field is canonically normalized and the vector field decouples from the inflaton. It is not difficult to check that power-law solutions with $h^{\prime} / h=$ const $V^{\prime} / V=\lambda=$ const cannot be found since the constraints $\epsilon \ll 1, \zeta \gg 1$, and $w>0$ (27) cannot be simultaneously satisfied. The same happens when considering a canonically normalized inflaton (15) coupled disformally to the vector via (61). This is consistent with the results of [27] where a detailed analysis is shown.

However, motivated by the DBI anisotropic solutions found in [20], we can slightly modify this Ansatz. Consider a DBI inflaton, with $P$ given as in (61), and a monomial, 
derivative coupling, $f$, as in (18). This could correspond to a model where the inflaton and the vector live in different D-branes. In [20] the authors found power-law anisotropic solutions with $h^{\prime} / h=$ const which implies that $\gamma=\gamma_{0}=$ const. Let us see this in some detail.

\section{A. DBI inflation with monomial, derivative coupling solutions}

Considering $h^{\prime} / h=$ const implies an exponential form for $h$, which we take as

$$
h(\phi)=\frac{h_{0}}{M_{\mathrm{Pl}}^{4}} e^{\frac{\Lambda}{M_{\mathrm{Pl}}} \phi} .
$$

Taking also an exponential form for the inflaton potential as before, (19), and power-law solutions for the scale factors and inflaton as in the canonically normalized case (20), we obtain the conditions

$\lambda \xi=-2, \quad \rho \xi+2 \zeta+2 \eta+2 n=1, \quad \Lambda=-\lambda$.

The requirement that $\Lambda=-\lambda$ is akin to setting $\gamma=\gamma_{0}=$ const. In terms of $h_{0}, \lambda$, and $\phi_{0} ; \gamma_{0}$ becomes

$$
\gamma_{0}=\left(1-4 \frac{h_{0}}{\lambda^{2}} e^{-\lambda \phi_{0}}\right)^{-\frac{1}{2}}
$$

By applying the exact same procedure of balancing the amplitudes as we used for the canonically normalized case (22)-(29), we obtain two analogous solutions that satisfy all of the system's equations:

$$
\zeta_{+}=\frac{D+\sqrt{E}}{72 n \lambda}, \quad \zeta_{-}=\frac{D-\sqrt{E}}{72 n \lambda},
$$

where

$$
D=6 \lambda+18 n \lambda-36 n^{2} \lambda+12 \rho+36 n \rho,
$$

and

$E=D^{2}-144 n \lambda\left(\frac{8}{\gamma_{0} \lambda}+\lambda-2 n \lambda+8 \rho-12 n \rho+12 \frac{\rho^{2}}{\lambda}\right)$.

Anisotropic inflationary solutions can now be found for suitable choices of the parameters, as long as they satisfy the constraints that $u, w$ [defined as before (23)] must be real and positive, and, of course, $\epsilon=1 / \zeta \ll 1$. As a concrete example, a stable solution can be found for $\lambda=0.01, \rho=300, n=-2, \gamma_{0}=1.5$. It has anisotropy $\Sigma / H=1.32254 \times 10^{-10}$ and slow-roll parameter $\epsilon=$ $3.33306 \times 10^{-5}$, making it compatible with data. We present in Appendix the stability analysis of this solution. Comparing to the canonically normalized case $\left(\gamma_{0}=1\right)$ discussed above, we can see the effect of the DBI kinetic term, which reduces very slightly the anisotropy and slow-roll parameter: $(\Sigma / H)_{c n}=2.97549 \times 10^{-10}$, $\epsilon_{c n}=4.99938 \times 10^{-5}$.

\section{DISCUSSION}

We have studied anisotropic inflationary solutions where the inflaton couples to a vector field derivatively. That is, the gauge kinetic function depends on both the inflaton and its derivative, $f(\phi, X)$, with $2 X=(\partial \phi)^{2}$. This coupling is motivated by D-brane inflationary models, where the D-brane features a vector on its world volume, and couples derivatively to the brane's position (or a Wilson line), the inflaton. Moreover, such couplings parametrize generic inflaton-matter couplings, which may be relevant in studies of the inflationary universe as a cosmological collider [15]. On the other hand, they also appear in the EFT of anisotropic inflation [16,17].

We started by presenting a general setup, which allows for the study of a wide range of models. We studied first an immediate generalization of the power-law anisotropic model studied in [22], where the gauge kinetic function is a monomial in $X,(18)$, while exponential in the inflaton. We found that there are no stable inflationary solutions for a purely shift symmetric coupling (that is $f_{\phi}=0$ ). However, stable derivative anisotropic solutions arise for a large range of parameters. Interestingly, compared to the nonderivative case, the derivative anisotropic solutions have a lower level of anisotropy. We presented two illustrative examples. In the most relevant from the observational point of view, the anisotropy goes down by 3 orders of magnitude with respect to the nonderivative case, $\Sigma / H=4 \times 10^{-6} \rightarrow$ $1 \times 10^{-9}$. We also found that the value of the anisotropy depends mildly on the power $n$ in (18), which needs to be negative. We also found that the DBI generalization of the power-law solutions in [22] can also be extended to the derivative case. That is, derivative anisotropic DBI solutions exist, where the gauge kinetic function is a monomial in $X$ [see (18)]. This example could correspond to a DBI inflationary model where the inflaton and the vector field live in different D-branes. On the other hand, in the case where the inflaton and vector live on the same brane, the gauge kinetic function is dictated by the model and given by (61). In this case, however, the requirements of inflation, small anisotropy, and a positive vector energy density $(w>0)$ are not compatible, and thus there are no solutions.

As we discussed in Sec. III, it is easy to understand the decrease in the anisotropy by looking at the final value of the anisotropy, which is given by the ratio of energy density of the vector field to that of the scalar field and given by (42), thus decreasing the level of anisotropy. A clear follow-up is to look at how the derivative coupling affects a potential anisotropy in the power spectra. We leave this for a future publication. 


\section{ACKNOWLEDGMENTS}

We thank Gianmassimo Tasinato for discussions. S. K. was supported by IKERBASQUE, the Basque Foundation for Science, and the Basque Government (IT-979-16), and Spanish Ministry MINECO (FPA2015-64041-C2-1P). I. Z. was partially supported by the STFC Grants No. ST/ N001419/1 and No. ST/L000369/1. S. K. would like to thank the Physics Department at Swansea University for warm hospitality and the College Research Fund for financial support. J.H. was supported by a Swansea College of Science Doctoral Training Centre (DTC) Research Scholarship funded partly by Swansea University and partly by the STFC.

\section{APPENDIX: STABILITY OF THE ANISOTROPIC DBI SOLUTIONS WITH DERIVATIVE COUPLINGS}

In this appendix we look at the stability of the anisotropic DBI solution discussed in the main text. We define dimensionless variables analogous to the canonically normalized case:

$W=\frac{\dot{\sigma}}{\dot{\alpha}}, \quad Y=\frac{\gamma_{0}}{M_{\mathrm{Pl}}} \frac{\dot{\phi}}{\dot{\alpha}}, \quad Z=\frac{f(\phi, X) e^{-\alpha+2 \sigma}}{M_{\mathrm{Pl}}} \frac{\dot{v}}{\dot{\alpha}}$

The Hamiltonian constraint (13) for this system becomes

$$
-\frac{V}{M_{\mathrm{Pl}}^{2} \dot{\alpha}^{2}}=3\left(W^{2}-1\right)+\frac{Y^{2}}{1+\gamma_{0}}+\frac{Z^{2}}{2}(1-4 n),
$$

and the slow-roll parameter

$$
\epsilon=3 W^{2}+\frac{Y^{2}}{2 \gamma_{0}}+\frac{Z^{2}}{3}(1-3 n)
$$

The equations of motion in terms of (A1) become

$$
\begin{gathered}
\frac{d W}{d \alpha}=\frac{1}{3} Z^{2}(W+1-3 n W)+W\left(3\left(W^{2}-1\right)+\frac{Y^{2}}{2 \gamma_{0}}\right) \\
\frac{d Y}{d \alpha}=F(Y, Z) Y\left\{3 \gamma_{0} Y^{4}+4 \gamma_{0} n Z^{2}\left[-3 W(3 W(4 n-1)+4)+Z^{2}(n(12 n-7)+1)-3\right]+2 Y^{2}\left[Z^{2}\left(\gamma_{0}^{2}-3 n\left(\gamma_{0}^{2}+4 n-1\right)\right)\right.\right. \\
\left.\left.+9 \gamma_{0}^{2} W^{2}-9\right]+3 \lambda Y\left(-4 n Z^{2}+6 W^{2}+Z^{2}-6\right)+6 \rho Y Z^{2}(1-4 n)+3 \gamma_{0} \lambda Y^{3}\right\} \\
\frac{d Z}{d \alpha}= \\
+Y(Y, Z) Z\left\{48 \gamma_{0} n^{3} Z^{4}+2 n\left[2 Z^{2} \gamma_{0}\left(-6-6 W+9 W^{2}+Z^{2}\right)+3 Y^{2}\left(-6+Z^{2}-\gamma_{0}^{2} Z^{2}\right)\right.\right. \\
\left.+3 \lambda Y\left(-6+6 W^{2}+Z^{2}\right)+3 \lambda \gamma_{0} Y^{3}\right]-4 n^{2} Z^{2}\left[\gamma_{0}\left(-18+36 W^{2}+7 Z^{2}\right)+6 Y(Y+\lambda)\right] \\
\left.+\gamma_{0} Y^{2}\left[2 \gamma_{0}\left(-6-6 W+9 W^{2}+Z^{2}\right)+3 Y(Y-2 \rho)\right]\right\}
\end{gathered}
$$

where

$$
F(Y, Z)=\frac{1}{6 \gamma_{0}\left(2 n Z^{2}(1-4 n)+\gamma_{0} Y^{2}\right)}
$$

This system reduces to the canonically normalized case (47)-(49) when $\gamma_{0} \rightarrow 1$. This system permits stable, anisotropic solutions. As an example, a stable solution can be found by taking $\lambda=0.01, \rho=300, n=-2, \gamma_{0}=1.5$. With these parameters, we find a fixed point at $(W, Y, Z)=$ $\left(1.32254 \times 10^{-10},-9.99917 \times 10^{-3}, \pm 3.45004 \times 10^{-5}\right)$. Linearization of Eqs. (A4)-(A6) around this fixed point yields

$$
\begin{aligned}
\frac{d \delta W}{d \alpha}= & -2.99997 \delta W-8.81622 \times 10^{-13} \delta Y \\
& \pm 2.30003 \times 10^{-5} \delta Z
\end{aligned}
$$

$$
\begin{aligned}
\frac{d \delta Y}{d \alpha}= & -1.2701 \times 10^{-6} \delta W-1.33371 \delta Y \\
& \pm 6.44229 \times 10^{-2} \delta Z
\end{aligned}
$$

$$
\begin{aligned}
\frac{d \delta Z}{d \alpha}= & \mp 6.90183 \times 10^{-5} \delta W \mp 2.53082 \times 10^{-2} \delta Y \\
& +8.89147 \times 10^{-4} \delta Z .
\end{aligned}
$$

This fixed point has eigenvalues $(-2.99997,-1.33249$, $\left.-3.33631 \times 10^{-4}\right)$ and is therefore stable. It has anisotropy $\Sigma / H=1.32254 \times 10^{-10}$ and slow-roll parameter $\epsilon=$ $3.33306 \times 10^{-5}$, making it compatible with data. 
[1] P. Ade et al. (Planck Collaboration), Planck 2015. XX. Constraints on inflation, Astron. Astrophys. 594, A20 (2016).

[2] P. A. R. Ade et al. (BICEP2 and Keck Array Collaborations), BICEP2/Keck Array VI: Improved Constraints on Cosmology and Foregrounds When Adding $95 \mathrm{GHz}$ Data from Keck Array, Phys. Rev. Lett. 116, 031302 (2016).

[3] J. Martin, C. Ringeval, R. Trotta, and V. Vennin, The best inflationary models after Planck, J. Cosmol. Astropart. Phys. 03 (2014) 039.

[4] J. Soda, Statistical anisotropy from anisotropic inflation, Classical Quantum Gravity 29, 083001 (2012).

[5] A. Maleknejad, M. M. Sheikh-Jabbari, and J. Soda, Gauge fields and inflation, Phys. Rep. 528, 161 (2013).

[6] R. Emami, Anisotropic inflation and cosmological observations, Ph.D. Thesis, Hong Kong University of Science and Technology, 2015.

[7] M.-a. Watanabe, S. Kanno, and J. Soda, Inflationary Universe with Anisotropic Hair, Phys. Rev. Lett. 102, 191302 (2009).

[8] K. Dimopoulos, Can a vector field be responsible for the curvature perturbation in the Universe?, Phys. Rev. D 74, 083502 (2006).

[9] K. Dimopoulos, M. Karciauskas, D. H. Lyth, and Y. Rodriguez, Statistical anisotropy of the curvature perturbation from vector field perturbations, J. Cosmol. Astropart. Phys. 05 (2009) 013.

[10] K. Dimopoulos, M. Karciauskas, and J. M. Wagstaff, Vector curvaton without instabilities, Phys. Lett. B 683, 298 (2010).

[11] K. Dimopoulos, Statistical anisotropy and the vector curvaton paradigm, Int. J. Mod. Phys. D 21, 1250023 (2012); Int. J. Mod. Phys. DErratum, 21, 1292003 (2012).

[12] K. Dimopoulos, D. Wills, and I. Zavala, Statistical anisotropy from vector curvaton in D-brane inflation, Nucl. Phys. B868, 120 (2013).

[13] A. Avgoustidis and I. Zavala, Warped Wilson line DBI inflation, J. Cosmol. Astropart. Phys. 01 (2009) 045.
[14] C. van de Bruck, T. Koivisto, and C. Longden, Disformally coupled inflation, J. Cosmol. Astropart. Phys. 03 (2016) 006.

[15] X. Chen, Y. Wang, and Z.-Z. Xianyu, Standard Model Background of the Cosmological Collider, Phys. Rev. Lett. 118, 261302 (2017).

[16] A. A. Abolhasani, M. Akhshik, R. Emami, and H. Firouzjahi, Primordial statistical anisotropies: The effective field theory approach, J. Cosmol. Astropart. Phys. 03 (2016) 020.

[17] T. Rostami, A. Karami, and H. Firouzjahi, Effective field theory of statistical anisotropies for primordial bispectrum and gravitational waves, J. Cosmol. Astropart. Phys. 06 (2017) 039.

[18] J. Kim and E. Komatsu, Limits on anisotropic inflation from the Planck data, Phys. Rev. D 88, 101301 (2013).

[19] A. Naruko, E. Komatsu, and M. Yamaguchi, Anisotropic inflation reexamined: Upper bound on broken rotational invariance during inflation, J. Cosmol. Astropart. Phys. 04 (2015) 045.

[20] T. Q. Do and W. F. Kao, Anisotropic power-law inflation for the Dirac-Born-Infeld theory, Phys. Rev. D 84, 123009 (2011).

[21] J. Ohashi, J. Soda, and S. Tsujikawa, Anisotropic power-law k-inflation, Phys. Rev. D 88, 103517 (2013).

[22] S. Kanno, J. Soda, and M.-a. Watanabe, Anisotropic powerlaw inflation, J. Cosmol. Astropart. Phys. 12 (2010) 024.

[23] A. Ito and J. Soda, Anisotropic constant-roll inflation, Eur. Phys. J. C 78, 55 (2018).

[24] T. Q. Do and W. F. Kao, Anisotropic power-law inflation for a conformal-violating Maxwell model, arXiv:1712.03755.

[25] M.-a. Watanabe, S. Kanno, and J. Soda, The nature of primordial fluctuations from anisotropic inflation, Prog. Theor. Phys. 123, 1041 (2010).

[26] E. Silverstein and D. Tong, Scalar speed limits and cosmology: Acceleration from D-cceleration, Phys. Rev. D 70, 103505 (2004).

[27] T. Koivisto and F. Urban, Disformal vectors and anisotropies on a warped brane, J. Cosmol. Astropart. Phys. 03 (2015) 003. 